\title{
Design of Barometer Hardware Based on Single-Chip Machine Caiyun Gui ${ }^{1}$ and Baobao Kang ${ }^{2}$
}

\author{
1. Yulin University, Yulin, 719000 \\ 2, Bureau of Geographical Prospecting INC., Zhuozhou, 07275)
}

Keywords: Barometer; Single-chip machine; Air pressure temperature sensor BMP085

\begin{abstract}
Barometer is an equipment that uses the pressure-sensitive components to directly convert the pressure to be measured into current or voltage signal, which is then processed and controlled by the single-chip machine for real-time display. The internal core element is the pressure sensor, which plays an important role in monitoring pressure, controlling pressure changes, and measuring physical parameters. The use of single-chip microcomputer for processing and control has a powerful control, small size, high integration and other advantages. In this design, first the pressure and temperature sensor BMP085 is used to collected the corresponding simulation signals of the environmental pressure and the temperature. This simulation signal is then processed by the signal conversion module within the pressure and temperature sensor. Finally, it is displayed on the LCD display module under the control of the STC89C51 single chip machine. In addition, the system can re-set the threshold by the key circuit and be stored in the memory module, and it can compared the actual value and the set threshold value to determine whether the actual value exceeds the limit before carrying out the sound and light alarm. It is verified through experiment that this design is safe, stable and economical, etc.
\end{abstract}

\section{Overall Scheme of the System}

Gather the pressure signal and temperature signal through the gas pressure sensor and the covert the analog and process other data; The integrated single-chip machine's main control is the 89C51 single-chip machine system, which consists of power supply circuit, clock circuit, reset circuit and other components, to achieve control functions; LCD display shows the current pressure value, AT24C02 storage chip storage threshold. When the collection pressure signal and the temperature exceeds the range value, the alarm circuit achieves the alarm function. System block diagram is as follows:

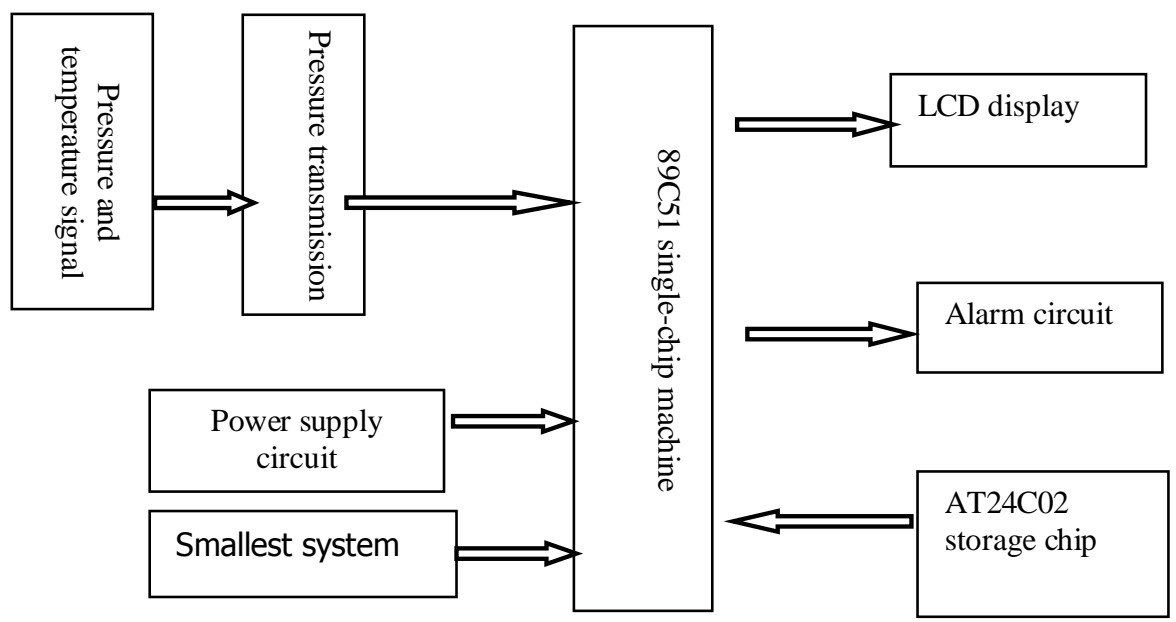

Figure. 1. System Chart 


\section{Design of Power Supply Circuit}

\section{Design Scheme}

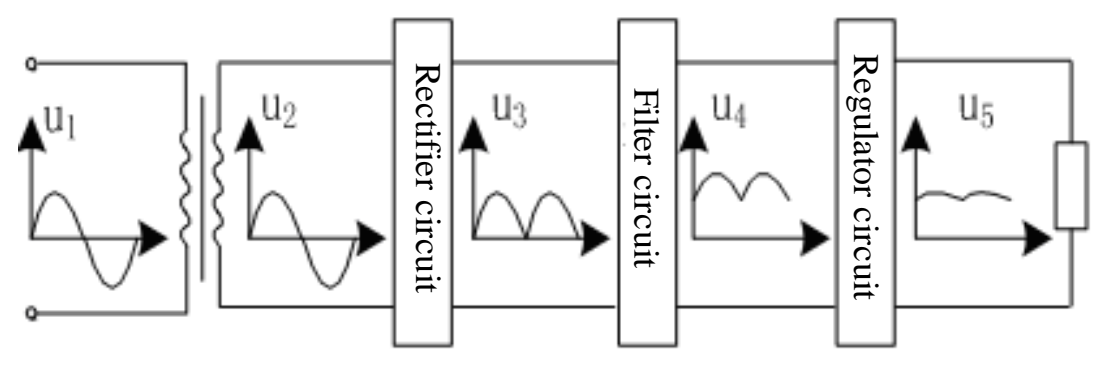

Figure 2. Design of Multi-output DC Voltage Regulator

The specific design is shown in Figure 2, the power transformer will be converted to mains voltage u1 $18 \mathrm{~V}$ AC voltage u2; Rectifier circuit will convert the AC voltage u2 pulsating DC voltage u3; filter circuit will pulsate DC voltage u3 into a smooth DC voltage u4; regulator circuit used to clear the fluctuations in the power grid and load changes to maintain the stability of the output voltage $\mathrm{u} 0$.

Design of Rectifying and Filtering Circuit. In the rectifier part, the circuit using silicon bridge rectifier KBU610 is used, and the average rectifier output current is 6A. The average rectified output voltage is:

$$
U_{3}=\frac{1}{2 \pi} \int_{0}^{2 \pi} u_{3} d(\omega t)=\frac{2 \sqrt{2} U_{2}}{\pi}=0.9 U_{2}
$$

Rectified output u3 Fourier series can be decomposed into:

$$
\begin{aligned}
& u_{3}=\sqrt{2} U_{2}\left(\frac{2}{\pi}-\frac{4}{3 \pi} \cos 2 \omega t-\frac{4}{15 \pi} \cos 4 \omega t\right. \\
& \left.-\frac{4}{35 \pi} \cos 6 \omega t \cdots\right)
\end{aligned}
$$

The pulsation coefficient $S$ is the ratio of the fundamental peak value to the average value, then the pulsation coefficient is:

$$
S=\frac{\frac{4 \sqrt{2} U_{2}}{3 \pi}}{\frac{2 \sqrt{2} U_{2}}{\pi}}=\frac{2}{3} \approx 0.67
$$

According to the Fourier transform of the output of the rectified u3, it can be seen that the AC voltage is rectified by the rectification circuit to output pulsating DC, in which both the DC component and the AC component are output. Filter circuit is to use the features that the voltage at both ends of the capacitor and the flow passing through the inductor cannot abruptly change to filter out the rectifier circuit output voltage AC components and maintain its DC components so as to achieve the purpose of smoothly outputting voltage waveform. 


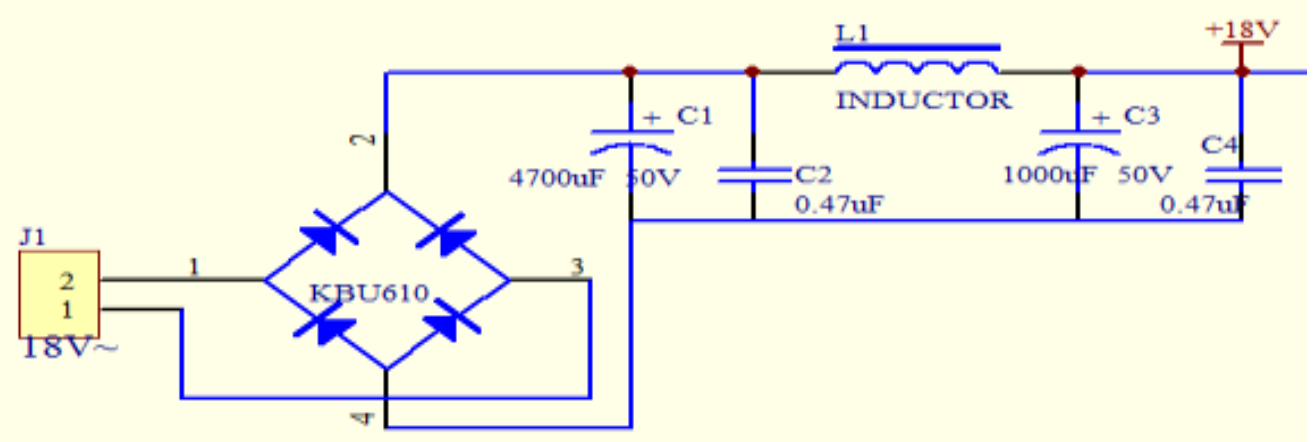

Figure 3. Rectifying and Filtering Circuit

The LC- $\pi$-type filter circuit is used as the filter circuit in this design. The circuit combines the advantages of inductive filtering and capacitive filtering, so it has the best wave filtering effect among all the passive filter circuits. Since the DC resistance of the inductor is small and the AC impedance is large, the DC component is basically not lost after passing the inductance. However, after the jwL voltage is divided, most of the DC voltage drops on the inductor, thus reducing the ripple component of the output voltage. The filter circuit has better filtering characteristics when the load current is larger or smaller, and thus has strong adaptability to the load, and is particularly suitable for situations where the current changes greatly. Specific circuit is shown in Figure 3.

Design of Voltage Stabilizing Circuit. Three-terminal regulator integrated circuit L7805 is used to realize the output of $+5 \mathrm{~V}$ voltage. Transformer secondary voltage U2's effective value is $18 \mathrm{~V}$, and after rectification and filtering, the value becomes $0.9 \mathrm{U} 2$. It passes two parallel cement resistor 5W50RJ, which is characterized by a good anti-overload capacity, and long-term high-temperature work. Input capacitor C9 is a bypass capacitor, the choice should be taken to ensure adequate pressure margin and the appropriate ripple current coefficient. The selected value in this design is $470 \mathrm{uF}$, and the voltage range is $50 \mathrm{~V}$. The output of the filter capacitor in parallel has the size of the way to filter out low-frequency and high-frequency interference signal. The maximum output current of each circuit is $1.5 \mathrm{~A}$. The specific circuit is shown in Figure 4.

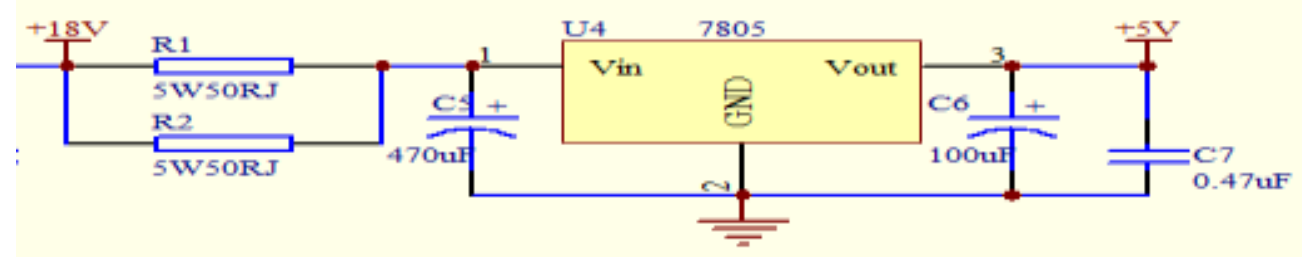

Fig4. +5V Voltage Regulator Output Circuit

\section{BMP085 and Single-Chip Machine's Interface Circuit}

The pin of the MBP085 is in the previous part, which has been described in detail. Its interface circuit with the single-chip machine is as shown in the following figure: 


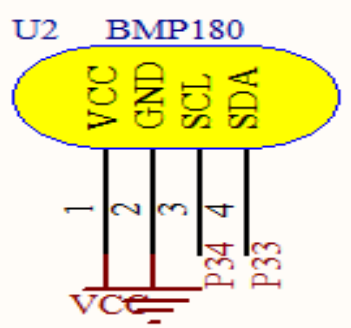

Figure 5. BMP085 and single-chip machine's interface circuit

\section{Design of Display Circuit}

Work principles of the LCD display: From the LCD structure, whether it is a laptop or desktop system, the LCD screen is composed of different parts of the hierarchical structure. The LCD consists of two glass plates, about $1 \mathrm{~mm}$ thick, separated by a uniform spacing of $5 \mu \mathrm{m}$ containing liquid crystal material. Since the liquid crystal material itself does not emit light, a lamp is provided on both sides of the display screen as a light source, and a backlight (or a light homogenizing plate) and a reflective film on the back of the liquid crystal display. The backlight is made of a fluorescent material that can emit light, and its role is to provide a uniform background light. Display circuit and microcontroller interface design is: P1.4 control LCD LCD1602 enable side, P1.1 control is the register selection side, P1.2 control is read and write strobe end, D0.0 D0.7 Connected to the liquid crystal display data cable. After single-chip STC89C51 detection and processing of digital signals, they are connected through the microcontroller port and the LCD data line is connected to the LCD display.

\section{Design of the Storage Module}

The AT24C02 is a 2K-bit serial CMOS E2PROM with 256 octets internal and CATALYST's advanced CMOS technology substantially reduces the device's power consumption. The AT24C02 has an 8-byte page write buffer. The device operates through the $\mathrm{I} 2 \mathrm{C}$ bus interface with a special write protection. AT24C02 in the circuit diagram with the microcontroller is shown in Figure 6.

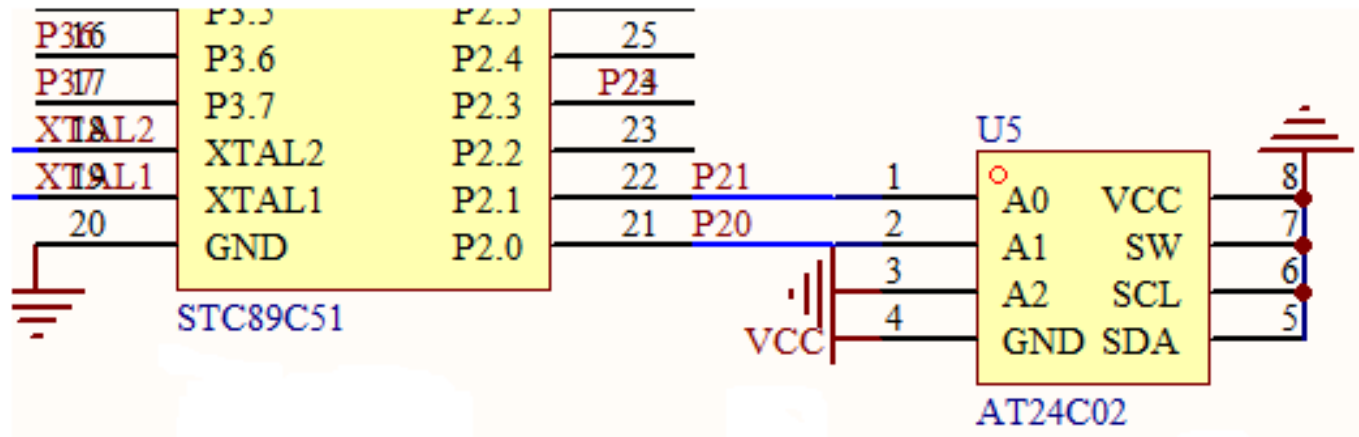

Figure 6. Diagram of Connection between AT24C02 and the Single-Chip Machine

\section{Conclusion}

This design mainly introduces the hardware circuit design principles of the barometer and gives the circuit design diagram of the main modules. With the 89C52 single-chop machine and BMP085 pressure and temperature sensor as the core, the pressure and temperature signals are converted and processed and then displayed on the LCD1602 LCD screen. Besides, threshold value is stored 
through the AT24C02 storage module. When the pressure and temperature signal exceeds the limit, the overrun sound and light alarm function is realized through the alarm circuit.

\section{References}

[1] Jin Hongli. Multi-channel Temperature Detection System based on STC89C52 and DS18B20 [J].Guide of Sci-tech Magazine, 2013,(17):83-84.

[2] Dragon Wanyi. Design of Digital Pressure Meter based on Wireless Transmission [D]. Shenyang: Shenyang University of Technology, 2013:11-13.

[3] Yang Ning, Hu Xuejun. Single-chip Machine and Control Technology [M]. Beijing: University of Aeronautics and Astronautics Press, 2005.

[4] Liu Wentao. Examples of the Application and Development of Single-Chip Machines [M]. Beijing: Tsinghua University press, 2005.

[5] Wang Shouzhong. Introduction to and Classical Examples of 51 Single-Chip Machine Development [M]. Beijing: People's Post and Telecommunications Press, 2007.

[6] Lei Furong, et al. Query Manual of 51 Single-Chip Machine's Common Module Design [M]. Beijing: Tsinghua University press, 2010. 